\title{
Conservation and characterization of unique porcine interstitial telomeric sequences
}

\author{
JI GuangZhen ${ }^{1}$, LIU Kai ${ }^{1}$, CHEN ChengBin ${ }^{1}$, RUAN WeiMin ${ }^{2}$, GLYTSOU Christina ${ }^{3}$, \\ YANG Yang ${ }^{1}$, OKUKA Maja ${ }^{4}$, SONG WenQing ${ }^{1}$, GAGOS Sarantis ${ }^{3}$, \\ LI Ning ${ }^{2} \&$ LIU Lin ${ }^{1 *}$

\footnotetext{
${ }^{1}$ State Key Laboratory of Medicinal Chemical Biology, College of Life Sciences, Nankai University, Tianjin 300071, China; ${ }^{3}$ Genetics Division, Center of Basic Research II, Biomedical Research Foundation of the Academy of Athens Greece, Athens 11527, Greece;
} \\ ${ }^{2}$ State Key Laboratory for Agrobiotechnology, College of Biological Sciences, China Agricultural University, Beijing 100193, China; \\ ${ }^{4}$ Department of Obstetrics and Gynecology, University of South Florida College of Medicine, Tampa, FL 33612, USA
}

Received September 8, 2012; accepted November 2, 2012

\begin{abstract}
Telomeres are composed of TTAGGG repeats and located at the ends of chromosomes. Telomeres protect chromosomes from instability in mammals, including mice and humans. Repetitive TTAGGG sequences are also found at intrachromosomal sites, where they are named as interstitial telomeric sequences (ITSs). Aberrant ITSs are implicated in chromosomal instability and found in cancer cells. Interestingly, in pigs, vertebrate telomere sequences TTAGGG (vITSs) are also localized at the centromeric region of chromosome 6 , in addition to the end of all chromosomes. Surprisingly, we found that botanic telomere sequences, TTTAGGG (bITSs), also localize with vITSs at the centromeric regions of pig chromosome 6 using telomere fluorescence in situ hybridization (FISH) and by comparisons between several species. Furthermore, the average lengths of vITSs are highly correlated with those of the terminal telomeres (TTS). Also, pig ITSs show a high incidence of telomere doublets, suggesting that pig ITSs might be unstable and dynamic. Together, our results show that pig cells maintain the conserved telomere sequences that are found at the ITSs from of plants and other vertebrates. Further understanding of the function and regulation of pig ITSs may provide new clues for evolution and chromosomal instability.
\end{abstract}

evolution, telomere, interstitial telomeric sequence (ITS), pig

Citation: Ji G Z, Liu K, Chen C B, et al. Conservation and characterization of unique porcine interstitial telomeric sequences. Sci China Life Sci, 2012, 55: 1029-1037, doi: 10.1007/s11427-012-4420-x

Telomeres are located at the ends of liner chromosomes, protecting chromosomes from instability [1]. The telomeric hexanucleotide (TTAGGG) ${ }_{n}$ repeat sequence is conserved in a wide range of species from invertebrates, including fish, amphibian and reptiles, to vertebrates, including mouse, human and pig [2], whereas the telomeric repeat of most plants is the heptanucleotide TTTAGGG [3,4]. The $(\mathrm{TTAGGG})_{n}$ repeat is also found at intrachromosomal sites,

*Corresponding author (email: liutelom@yahoo.com) including those repeats located close to the centromeres, and at interstitial sites, namely, the sites between the centromeres and telomeres, in which case the repeats are called interstitial telomeric sequences (ITSs) [5-7]. ITSs have been described in many species, including fish [8-11], chicken [12], mouse [13,14], Chinese hamster [15,16], primates [17], Arabidopsis [18] wheat [19], and human [20,21]. ITSs might originate from ancestral intrachromosomal rearrangements (inversions and fusions), from differential crossing-over or from the repair of double-strand break 
during karyotype and genome evolution [7,20,22-24]. Based on their sequence organization and genomic location, two types of ITSs have been identified: type I. heterochromatic ITSs (het-ITSs), with large (up to hundreds of kb) stretches of telomeric-like DNA localized mainly at centromeres; type II. short ITSs (s-ITSs), with short stretches of telomeric hexamers distributed at the internal sites of the chromosomes [25]. Het-ITSs have only been described in some vertebrate species and might represent remnants of evolutionary chromosomal rearrangements, whereas s-ITSs are probably present in all mammalian genomes [25].

The origin and function of ITSs are still of interest in biomedicine. ITS loci are polymorphic and stretches of exact internal telomeric repeats can be highly unstable, like microsatellites [26]. In sporadic gastric tumors, ITS loci with both microsatellite instability (MSI) and without MSI in their chromosome are unstable [26]. Cytogenetic studies showed a relationship between the location of ITSs and fragile sites [17]. Telomere-associated proteins also bind to ITS region, and the reported presence of TRF1 and TRF2 at ITS loci suggest that these proteins might favor $\mathrm{Ku}$ dependent non-homologous end-joining of double-strand breaks [27,28]. Recently, the binding of human telomeric proteins to ITS was found to influence gene transcription [29,30].

Pig ITSs sequences that are homologous to segments of human chromosomes 1 and 19 were found to be located approximately at the centromeric region of chromosome 6 [31]. These ITS, therefore, can be considered as het-ITSs. We aimed to further characterize the origin and length regulation of ITSs using pig cells as a model, because, in pigs, ITSs have only been found at the centromeric region of chromosome 6. With the exception of nematodes and arthropods, the (TTAGGG) ${ }_{n}$ repeat is conserved in most Metazoa [2]. Unexpectedly, we found both vertebrate and botanic telomeric sequences (bITSs and vITSs) at ITS of pig chromosome 6. This novel finding of plant (TTTAGGG) telomeric repeats in mammal may provide new clues into the involvement of telomeres in species evolution and a possible interpretation for the specific characteristics of pig telomeres. Furthermore, we show that the lengths of vITSs are associated with the length of terminal telomeres (TTSs) and are dynamic and unstable.

\section{Materials and methods}

\subsection{Cell isolation and culture}

The use of the animals for this research was approved by the Institutional Animal Care and Use Committee at Nankai University, China. Mouse embryonic fibroblasts isolated from our laboratory and routinely stored in liquid nitrogen were also used for comparison of telomere length. Fibroblasts from fetal (embryonic day 50), newborn (8 days after birth), and adult (3-4 months old) Yorkshire pigs were isolated by a standard tissue-attachment method used in our laboratory. Briefly the tissues were peeled, minced into 1 $\mathrm{mm} \times 1 \mathrm{~mm}$ pieces, placed onto culture dishes, and incubated in Dulbecco's Modified Eagle Medium (DMEM) (GIBCO, Invitrogen, Carlsbad, CA, USA), containing $10 \%$ fetal bovine serum (FBS) (Thermo Scientific, San Diego, USA). Gelatinous bone marrow from femurs and tibias and of fetal and newborn piglets was extracted under sterile conditions. The bones were rinsed with DMEM after both ends were cut. The recovered cells were centrifuged, resuspended, and plated onto culture dishes. DMEM, with $15 \%$ FBS, was changed every other day. Cells were passaged every 3 or 4 days until the cells reached $90 \%$ confluence. Fibroblasts from Taihu pigs were provided by Lai LiangXue from Guangzhou Institute of Biomedicine and Health, Chinese Academy of Sciences.

Pig induced pluripotent stem (iPS) cell lines (JN1 and JN2) were generated from mesenchymal cells by transduction with four Yanamaka factors [32]. Cells were cultured with DMEM medium with $15 \%$ FBS. Then, $5 \times 10^{4}$ cells per 6-well plate were transduced with pMXs-based retroviral vectors (pMXs-Sox2, Klf4, Oct4, c-Myc). After infection, the cells were replated in $10 \mathrm{~mL}$ iPS medium containing knock-out DMEM medium (Invitrogen, CA, USA) added with $20 \%$ FBS, $1000 \mathrm{U} \mathrm{mL}^{-1} \mathrm{LIF}, 4 \mathrm{ng} \mathrm{mL} \mathrm{m}^{-1} \mathrm{bFGF}$ (Invi-

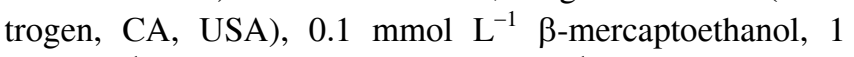
mmol L ${ }^{-1}$ L-glutamine and $0.1 \mathrm{mmol} \mathrm{L}^{-1}$ nonessential amino acids added. Three days after infection, the cells were passaged on mouse embryonic fibroblast (MEF) feeders and the medium was changed every day. Embryonic stem cell (ESC)-like colonies were picked and passaged using standard protocols.

\subsection{Chromosome spreads and telomere quantitative fluorescence in situ hybridization (Q-FISH)}

Cells including pig and mouse were incubated with $0.2 \mu \mathrm{g}$ $\mathrm{mL}^{-1}$ nocodazole (Sigma, St. Louis, MO, USA) for 3-5 h to enrich the cells at metaphase. Chromosome spreads for the different cells were made using a routine method. The metaphase-enriched cells were exposed to hypotonic treatment with $75 \mathrm{mmol} \mathrm{L}{ }^{-1} \mathrm{KCl}$ solution, fixed with methanol:glacial acetic acid (3:1) and spread onto clean slides. Telomere FISH and quantification were performed as described previously [33], except that 5'FAM-(TTTAGGG) $)_{3}$ and 5'TAMRA-(TTAGGG) ${ }_{3}$ DNA probes were used for the detection of telomeres, and fluorescence isothiocyanate (FITC)-labeled (CCCTAA) 3 peptide nucleic acid (PNA) probe was used for quantification of telomeres. Telomeres were denatured at $80^{\circ} \mathrm{C}$ for $3 \mathrm{~min}$ and hybridized with the telomere PNA probe $\left(0.5 \mu \mathrm{gL}^{-1}\right)$ (Panagene, Daejeon, Korea). Chromosomes were stained with $0.5 \mu \mathrm{g} \mathrm{mL}{ }^{-1}$ DAPI. Fluorescence from chromosomes and telomeres was digi- 
tally imaged on a Zeiss microscope with FITC/DAPI filter sets, using AxioCam and AxioVision software 4.6 (Carl Zeiss, Germany). For quantitative measurement of telomere length, telomere fluorescence intensity was integrated using the TFL-TELO program (kindly gifted by P. Lansdorp, Terry Fox Laboratory, Vancouver, Canada). More than 30 metaphase spreads from each cell line were examined.

\subsection{Telomere chromatid orientation (CO)-FISH}

Strand specific CO-FISH was performed according to Bailey et al. [34], with minor modifications. Sub-confluent cell monolayers were incubated with 5'-bromo-2'-deoxyuridine (BrdU) for $8 \mathrm{~h}$. Nocodazole was added for the last four hours prior to cell harvest and metaphase spreads were prepared by standard cytogenetic method as described above. Chromosome preparations were stained with Hoechst 33258 $\left(0.5 \mu \mathrm{g} \mathrm{mL}^{-1}\right)$, incubated in $2 \times \mathrm{SSC}$ (Invitrogen) for $20 \mathrm{~min}$ at room temperature (RT) and exposed to $365-\mathrm{nm}$ UV light (Stratalinker $1800 \mathrm{UV}$ irradiator) for $40 \mathrm{~min}$. The 5'-bromo2'-deoxyuridine-substituted DNA was digested with exonuclease III (Promega). The slides were then dehydrated through cold ethanol series and air-dried. PNA-FISH was performed with fluorescein-OO-(CCCTAA) ${ }_{3}$ (Bio-Synthesis Inc, Texas USA). Slides were hybridized, washed, dehydrated, mounted and counterstained with VectaShield antifade medium (Vector), containing $0.1 \mu \mathrm{g} \mathrm{mL} \mathrm{m}^{-1}$ DAPI. Digital images were captured using a CCD camera on a Zeiss Axio-Imager Z1 microscope equipped with Metasystems Isis software (Carl Zeiss, Germany).

\subsection{Plant chromosome preparation and FISH}

Root tips of Horsebean (Vicia faba Linn) and Hyacinthaceae (Hyacinthus orientalis L.) were treated in $4 \mu \mathrm{mol} \mathrm{L} \mathrm{L}^{-1}$ amiprophos-methyl (APM) for $3 \mathrm{~h}$. Root tips were digested in an enzyme mixture including $2.5 \%(\mathrm{w} / \mathrm{v})$ cellulase and pectinase for $1 \mathrm{~h}$, double distilled water for $20 \mathrm{~min}$ and Carnoy's fixation (3:1, methanol:acetic acid, v/v). Finally, root tips were smeared on a slide and dried with hot air. Slides with metaphase spread chromosomes were treated with $70 \%$ deionized formamide in $2 \times \mathrm{SSC}$ at $70^{\circ} \mathrm{C}$ for $2 \mathrm{~min}$ and dehydrated in a $-20^{\circ} \mathrm{C}$ ethanol series $(70 \%, 85 \%$, and $100 \%$ ethanol; 5 min each). Ten microliters of hybridization mixture was applied to each slide and sealed under a coverslip $(18 \mathrm{~mm} \times 18 \mathrm{~mm})$ with rubber cement. After overnight incubation the coverslips were removed and the slides were washed at room temperature 10 min with $4 \times$ $\mathrm{SSC} / 0.2 \%$ Tween 20 at $37^{\circ} \mathrm{C}$. The slides were then mounted in antifade solution (Vectashield) with DAPI.

\subsection{Statistical analysis}

Linear relationships and regression analyses were performed using SigmaPlot 8.0 (Systat Software, San Jose, CA,
USA). Significant differences were defined as $P<0.05,0.01$, or lower.

\section{Results}

\subsection{Both vertebrate and botanic telomeric sequences were found at the interstitial region of pig chromosome 6}

Chromosome spreads of various pig cells were analyzed by G-banding, following FISH using 5'-FAM-(TTTAGGG) (botanic telomeric sequences, bITSs) and 5'-TAMRA(TTAGGG) ${ }_{3}$ (vertebrate telomeric sequences, vITSs) DNA probes. We found that vITSs (TTAGGG) ${ }_{n}$ repeats were hybridized at the terminal and interstitial regions (Figure 1B), while bITSs (TTTAGGG) $)_{n}$ were only at the interstitial region of pig chromosome 6 (Figure 1A). High-resolution FISH and G-banding allowed the arrangement of bands in the long arm of chromosome 6 to be specified (Figure $1 \mathrm{C}-\mathrm{E}$ ); vITSs were likely at q21-22 bands of chromosomes 6 as previously shown [31,35], and bITSs were mapped to the q22-23 bands of the same chromosome (Figure 1E). Although the vITSs and bITSs partial overlap, they were still discernible (Figure 1E). Most bITSs are (TTTAGGG) repeats $[3,4]$, whereas vertebrate $(\mathrm{TTAGGG})_{n}$ repeat occasionally appears in plants [4], such as some members of the Asphodelaceae family [36,37] and Arabidopsis [38]. These differences presumably could result from a mutation in the template region of telomerase [39]. Although, thus far, plant (TTTAGGG) $)_{n}$ sequences had never been reported in Metazoa $[2,40]$, we identified bITSs in domestic pigs that again co-localized with vITS (Figure 2). Thus, for the first time, plant telomeric (TTTAGGG) $)_{n}$ repeats have been observed in pig genomes.

\subsection{Pig telomeres share vertebrate and plant charac- teristics}

Chromosomes spreads of horsebean, members of the Hyacinthaceae family (Asparagales order), mouse and pig were hybridized with the 5'-FAM-(TTTAGGG) $)_{3}$ and 5'TAMRA-(TTAGGG) ${ }_{3}$ DNA probes. We found that most of the plant telomeres exhibited Arabidopsis-type telomeric sequence $\left(\right.$ TTTAGGG) ${ }_{n}$ repeats with no vertebrate (TTAGGG) $)_{n}$ repeats, for example, Canavalia ensiformis (horsebean) (Figure 3A). In addition, telomere doublets were found in horsebean. However, we found both bITS and vITS repeats in members of the Hyacinthaceae family, the signals for the vertebrate telomere (TTAGGG) ${ }_{n}$ repeats were much stronger at the ITSs than at the TTSs (Figure 3B). In contrast, normal mammalian cells seemed to have very few telomere doublets; took mouse for example, telomere doublets were rarely found at TTSs (Figure 3C). Moreover, no vITSs were found in mouse telomeres, although both bITS and vITS were detected in pig cells. We 

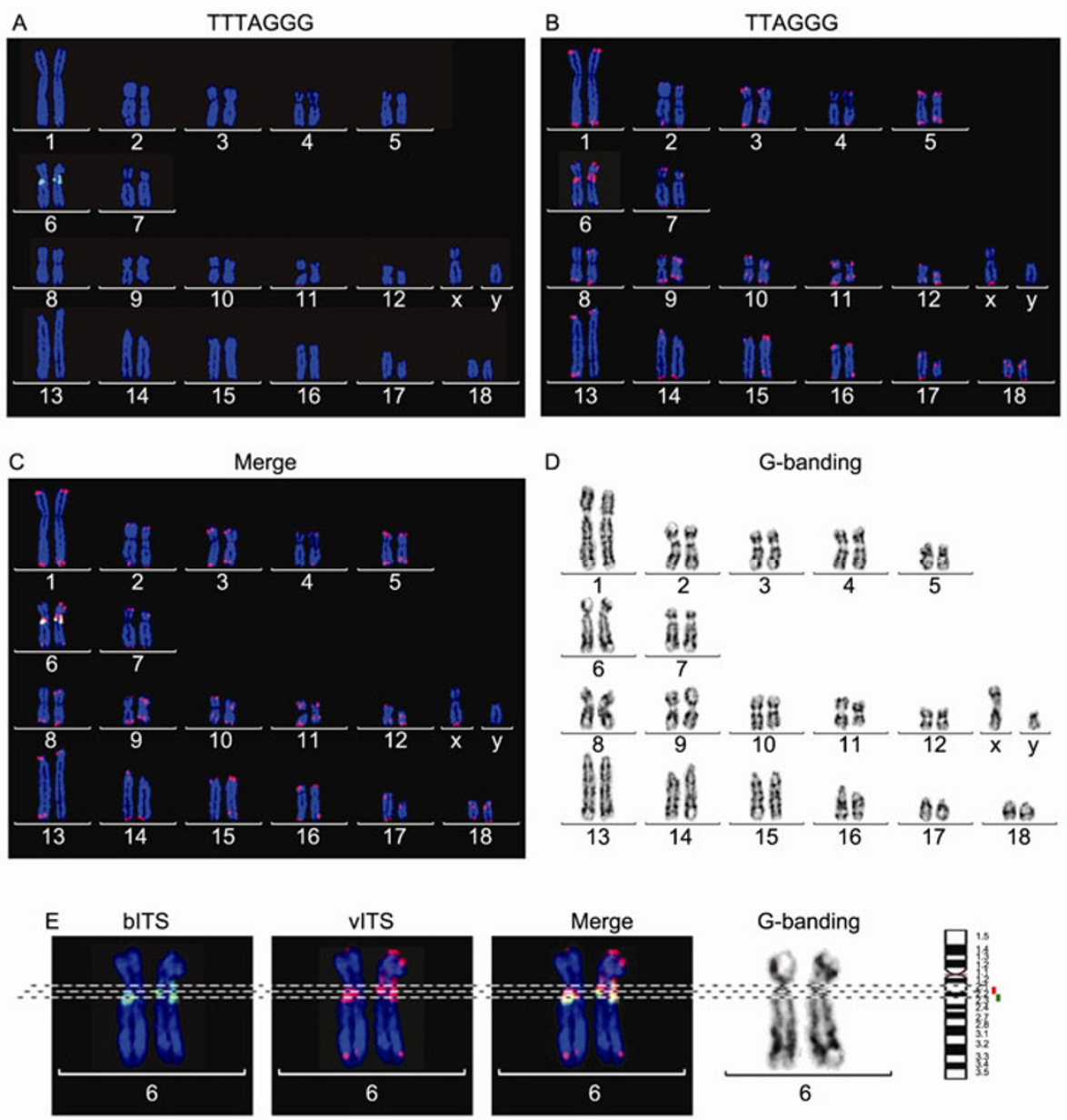

Figure 1 Plant and vertebrate telomeric sequences found at the interstitial region of pig chromosome 6. Metaphase spreads were analyzed by G-banding, following FISH with FAM-(TTTAGGG) ${ }_{3}$ and TAMRA-(TTAGGG) $)_{3}$ DNA probes. A, FISH with the botanic telomeric probe, $5^{\prime}$ FAM-(TTTAGGG) $)_{3}$. Green, botanic telomeric sequences; B, FISH with the vertebrate telomeric probe, 5'TAMRA-(TTAGGG) $)_{3}$. Red, vertebrate telomeric sequences. C, Merged of image of $1 \mathrm{~A}$ and $1 \mathrm{~B}$. Green dots, botanic telomeric sequences; red dots, vertebrate telomeric sequences; blue, DAPI-stained chromosomes. D, Representative G-banding analysis of pig karyotype corresponding to the FISH analysis in Figure 1A. E, High resolution of chromosome 6 showing the position of botanic interstitial telomeric sequences (bITSs) and vertebrate interstitial telomeric sequences (vITSs). Broken lines indicate the positions of bITSs and vITSs; bITS at approximately q22-23 (green bar) and vITS at q21-22 (red bar).
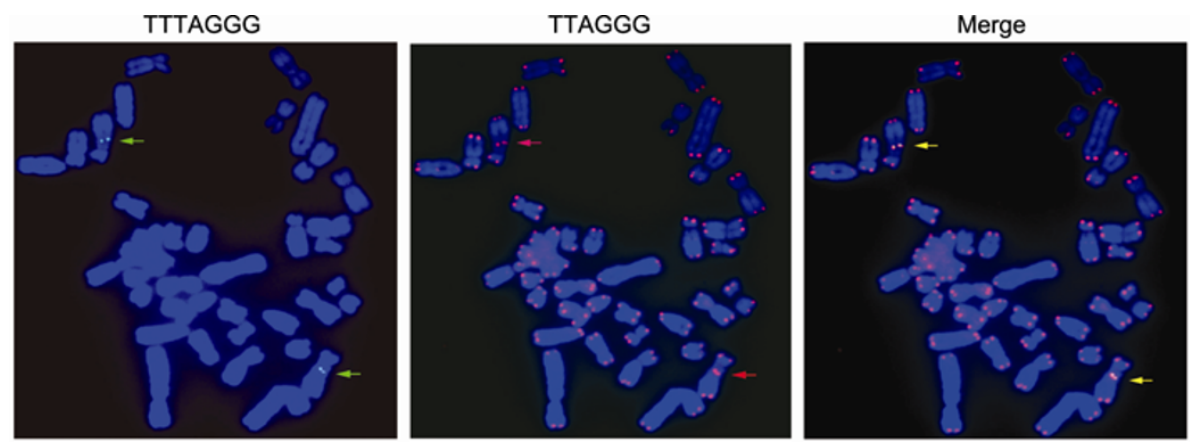

Figure 2 Metaphase spreads of plant and vertebrate telomeric sequences at the interstitial region in Taihu pig fibroblasts. The spreads were analyzed by FISH using FAM- (TTTAGGG) ${ }_{3}$ (green) and TAMRA-(TTAGGG) ${ }_{3}$ DNA (red) probes.

also observed telomere doublets at pig telomeres (Figure 3D). These findings suggest that, in botanic telomere evolution, a switch-point might have occurred when the Arabidopsis-type telomere was replaced by the typical verte- brate telomere in Asparagales [36,37]. The identification of bITSs in pig telomeres suggests that plant and vertebrate telomeres share certain characteristics, perhaps implicating telomeres in evolution. 

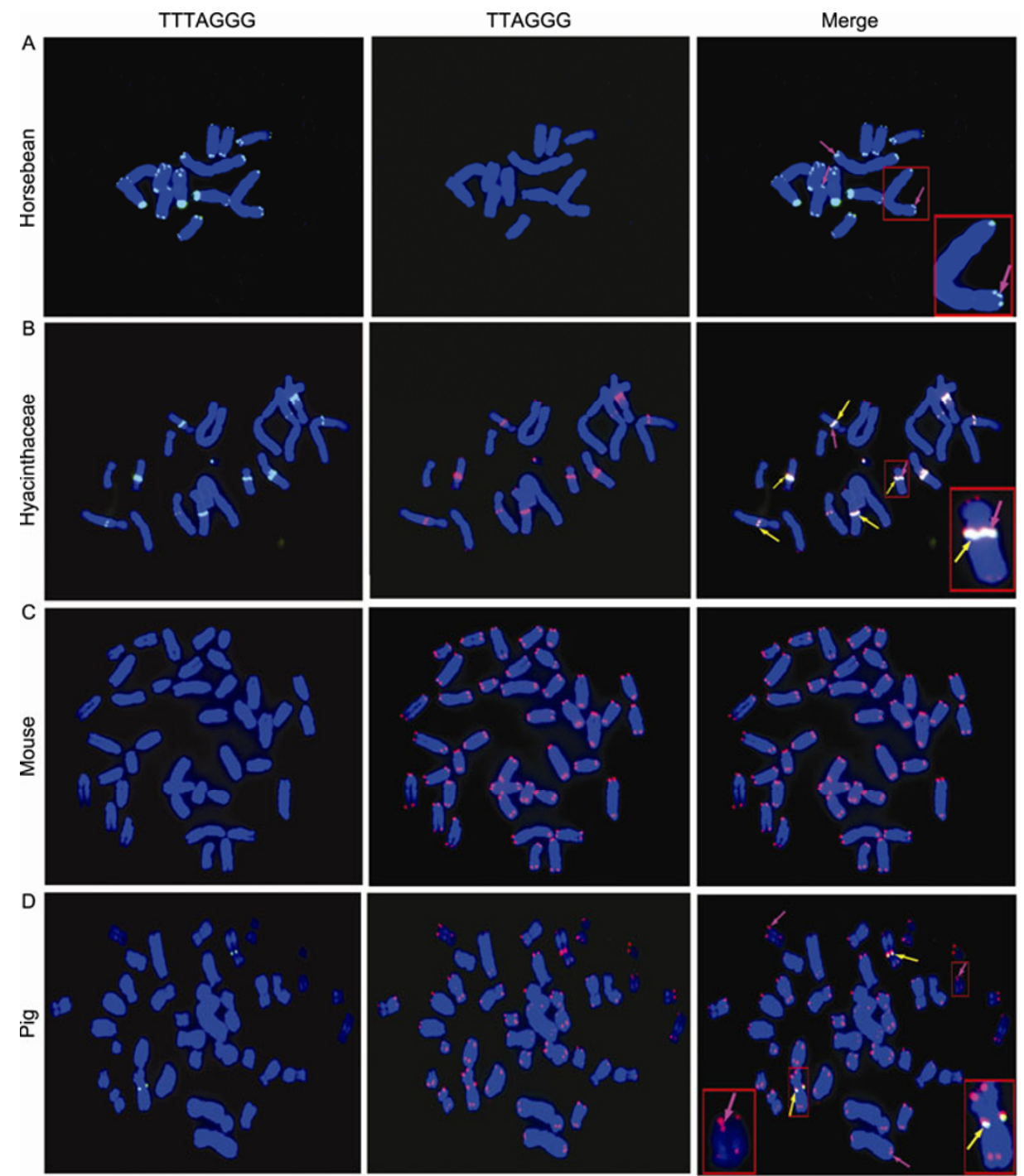

Figure 3 Pig telomeres share characteristics of plant and vertebrate telomeres. Chromosomes spreads were hybridized with $5^{\prime}$ FAM-(TTTAGGG) ${ }_{3}$ and 5'TAMRA-(TTAGGG) ${ }_{3}$ DNA probes. A, Chromosome spread of horsebean, showing the Arabidopsis-type of telomeric (TTTAGGG) ${ }_{n}$ repeats at the end of chromosomes. B, Chromosome spread of the Hyacinthaceae family. Typical terminal telomeres are not obvious at the end of chromosomes; however, both bITSs and vITSs appear at interstitial loci and telomere doublets can also be seen. C, Chromosome spread of representative telomeres of mouse embryonic fibroblast. The (TTAGGG) $)_{3}$ probes were positively hybridized at the end of chromosomes. D, Chromosome spread of typical telomeres of pig. The $(\text { TTAGGG })_{n}$ repeats are found at the ends and at the interstitial region of chromosomes. Repetitive (TTTAGGG) ${ }_{n}$ repeats found only at the interstitial centromeric region of the chromosomes. Green dots, botanic telomeric sequences; red dots, vertebrate telomeric sequences; blue, DAPI-stained chromosomes; yellow arrows, ITSs of the vertebrate and plant; pink arrows, telomere doublets.

\subsection{Dynamics of vITSs in association with TTSs}

Correlation analysis of vertebrate TTSs and vITSs by Q-FISH found that the lengths of vITSs varied and that the vITS length variations were highly correlated with the lengths of TTSs of various pig cell types (Figure 4). Polymorphisms in vITSs were also detected in the pig cells. The length of the pig vITSs ranged from 15.8 to 46.35 telomere fluorescence units (T.F.U.) (about 11.7-34.2 kb, estimated by Telomere Restriction Fragment (TRF)), in fetal fibroblast. Pig ITSs were longer than human ITSs with about 500-700 bp, and shorter than hamster ITSs with 250-500 $\mathrm{kb}$ [16]. The ITSs were, on average, longer than the TTSs in the same pig cell type (Figure 4), but seemed to share the same length dynamics as TTSs. The average lengths of both ITSs and TTSs were analyzed by Q-FISH for 17 cell lines, including fibroblasts and mesenchymal cells from different individuals and iPS cells during subculture. During subculture of three normal cell lines, fetal mesenchymal cells (FM), newborn mesenchymal cells (NM) and adult fibroblast cells (AF), the length of vITSs decreased at later passages (Figure $4 \mathrm{~A}-\mathrm{C}$ ). However, the length of vITSs generally increased in two iPS cell lines induced from the same progenitors NM at passage 4 (Figure 4D-E). The lengths of the vITSs changed with the lengths of the TTSs (Figure 4D-E). Five cell lines from three individual pigs were 

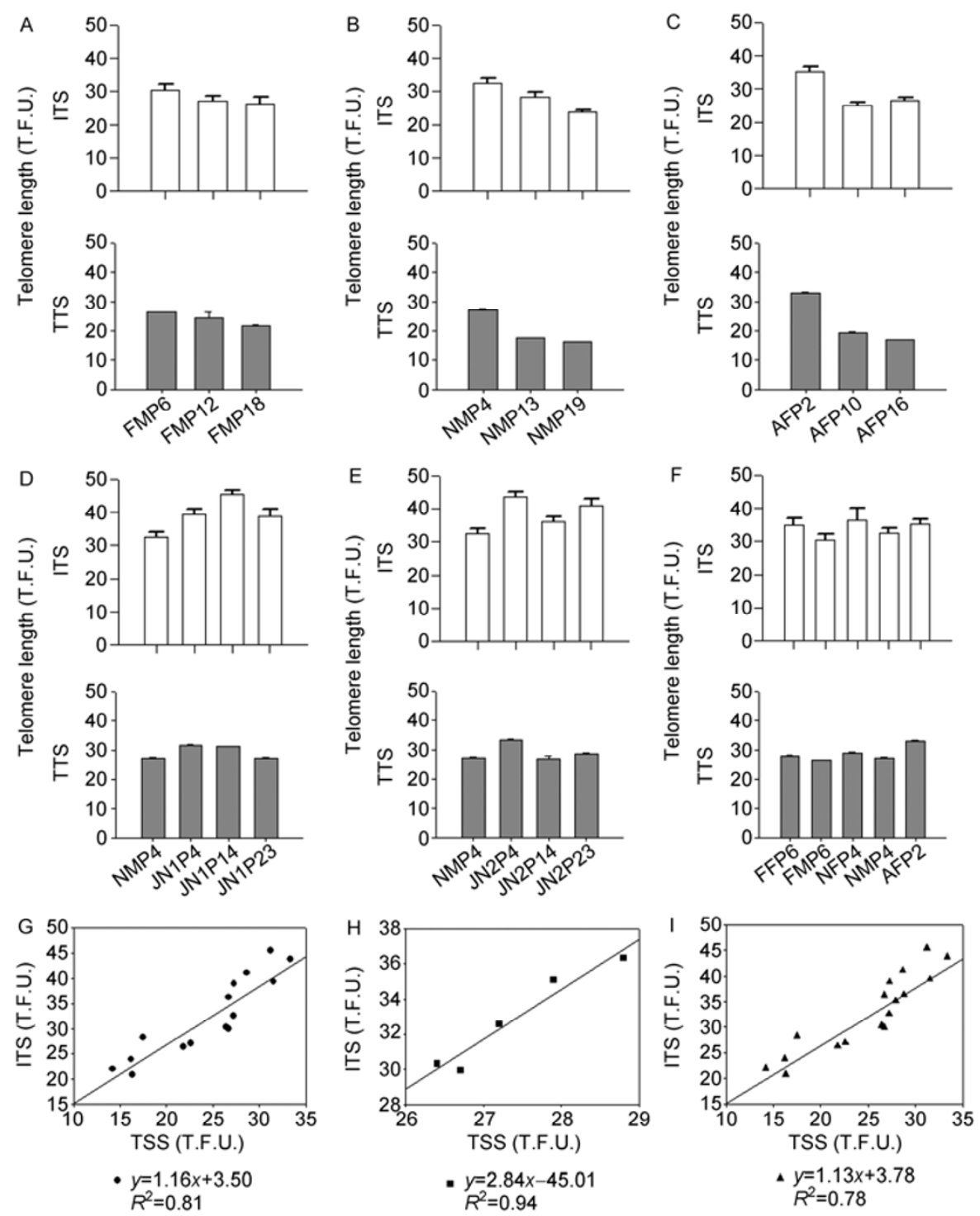

Figure 4 Correlation analyses of vertebrate terminal telomeric sequences and interstitial telomeric sequences by Q-FISH. A, Telomere length of fetal mesenchymal cells (FM) during subculture. FMP6, 12, 18, fetal mesenchymal cells at passage 6, 12 or 18. Mean telomere length is shown as mean T.F.U. \pm SD. B, Telomere length of newborn mesenchymal cells (NM) during subculture. NMP4, 13, 19, newborn mesenchymal cells at passage 4, 13 or 19. C, Telomere length of adult fibroblast cells (AF) during subculture. AFP2, 10, 16, adult fibroblast cells at passage 2, 10 or 16. D and E, Telomere length of induced pluripotent stem cells (iPS, named JN cell lines) generated from new born mesenchymal cells at passage 4 (NMP4). Telomere lengths of JN1 (4D) and JN2 (4E) at passage 4,14 or 23. F, Telomere lengths of vITS and TTS in different cell lines at early passages. FFP6, fetal fibroblasts at passage 6; FMP6, fetal mesenchymal cells at passage 6; NFP4, newborn fibroblasts at passage 4; NMP4, newborn mesenchymal cells at passage 4; AFP2, adult fibroblasts at passage 2. $\mathrm{G}$, Regression analysis of vITSs and TTSs of various cell lines at different passages. H, Correlation of telomere length between TTSs and ITSs from different individuals. I, Correlation of telomere length between the vITS and TTS of all samples. $P<0.001$. T.F.U., telomeric fluorescence unit.

directly compared and again the length of the vITSs changed with the TTS lengths (Figure 4F). The ITSs were found to be highly correlated with the TTSs by regression analysis (Figure 4G-I). Together, pig ITSs are dynamic and highly correlated with the TTSs.

\subsection{Telomere doublets and telomere sister chromatid exchange (T-SCE) at the interstitial region}

We analyzed rates of T-SCE by CO-FISH $[34,41]$. The nascent DNA strand was labeled with BrdU and specially digested. The sister chromatid with the weaker telomere signals indicative of T-SCE could be observed in metaphase spreads. We selected a few pig cell types (newborn fibroblasts, newborn mesenchymal cells and iPS cells) for the T-SCEs analysis. The frequency of T-SCEs was generally high in the TTSs of pig cells (Figure 5A-D). Similarly, T-SCE was also found in the interstitial region of chromosome 6 albeit at a lower frequency than in the TTSs (Figure $5 \mathrm{C})$. 

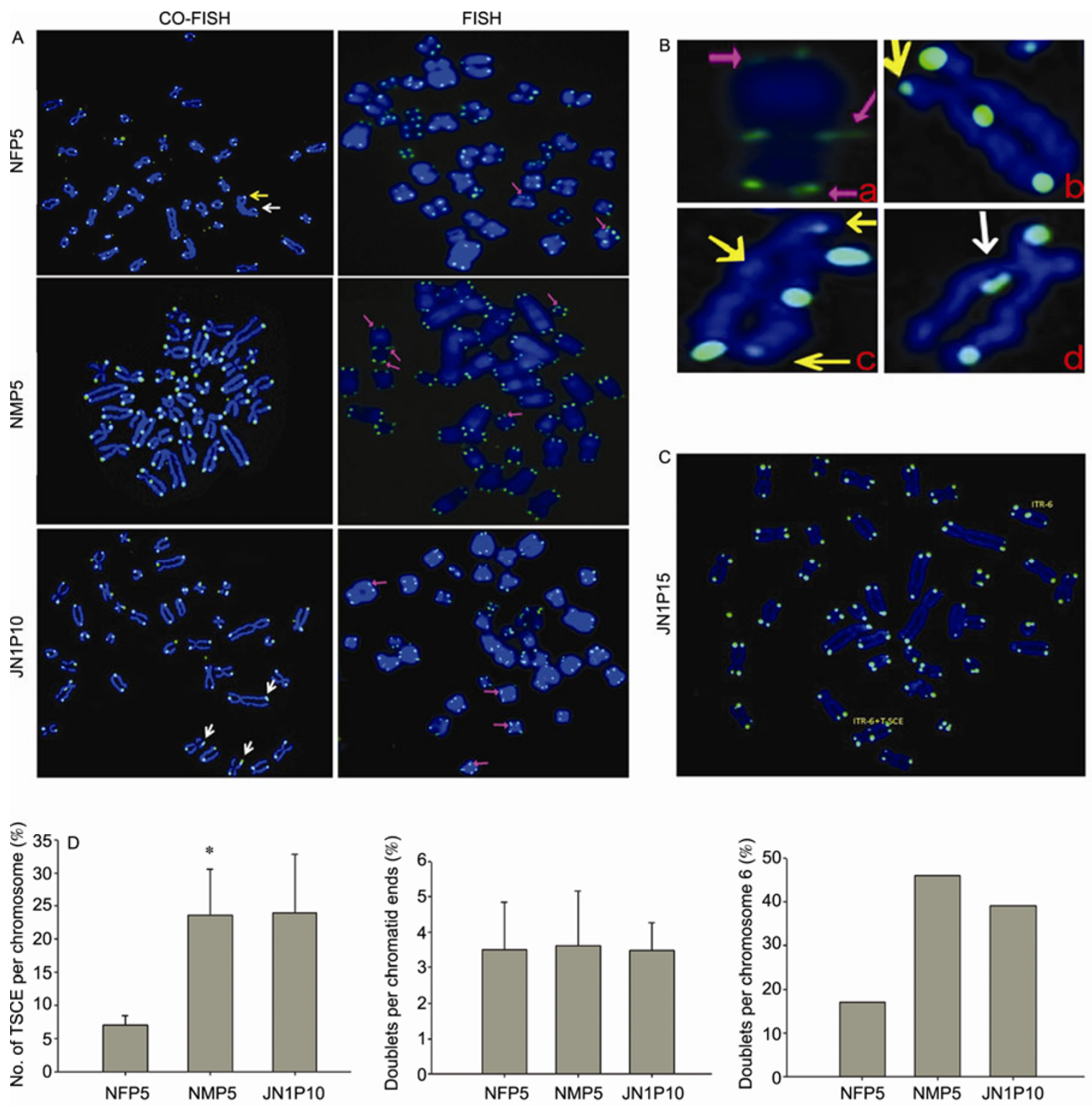

Figure 5 Telomere sister chromatid exchange (T-SCE) and doublets by CO-FISH and Q-FISH. A, T-SCE at TTSs of NFP5, NMP5 and JN1P10. Telomere doublets in NFP5, NMP5 and JN1P10 at both terminal and interstitial positions revealed by FISH with peptide nucleic acid probes (PNA). Blue, DAPI-stained chromosomes; green dots, vertebrate telomeric sequences; yellow arrowheads, T-SCEs; white arrowheads, doublets after BrdU incorporation; purple arrowheads, doublets. B, Enlarged images showing single pig chromosome 6 from 5A. a, representative doublets at interstitial region detected by FISH (purple arrowheads); b and c, typical T-SCE at terminal region (yellow arrowheads) and interstitial region detected by CO-FISH; d, doublets at interstitial region by CO-FISH (white arrowheads). C, Typical T-SCE at ITS loci by CO-FISH in pig iPS cells. D, Frequency (\%) of TSCE per chromosomes.

$*, P<0.05$, compared with NFP5. E, Frequency of telomere doublets per chromatid ends. F, Frequency of telomere doublets in ITSs per pig chromosome 6.

The telomere doublets were consistently detected at both TTSs and ITSs in pig cells (Figure 5A, B, E, F) and the doublets still existed at the ITSs after digestion and hybridization (Figure 5B). Telomere doublets could indicate instability of ITSs and TTSs.

\section{Discussion}

In this study, we showed vITSs and bITSs are present at the centromeric loci of chromosome 6 in pig cells. Furthermore, we demonstrated that the length of ITSs is not fixed, but rather it is dynamic. Moreover, dynamics in lengths of vITS are correlated with that of TTSs.

During evolution, (TTAGGG) ${ }_{n}$ repeats have been conserved in Metazoa with the exception of Nematoda and Arthropoda that have (TTAGC) ${ }_{n}$ and (TTAGG) ${ }_{n}$ repeats, respectively [2]. Pig telomeres had conserved (TTAGGG) repeats as reported in other vertebrates [31,42]. Most plants have (TTTAGGG) ${ }_{n}$ repeats as found, for example, in Arabidopsis telomeres $[3,4]$. In the monocot plant Asparagales order, the Arabidopsis-like telomere (TTTAGGG) ${ }_{n}$ repeats 
switched to human telomeric (TTAGGG) ${ }_{n}$ repeats [4]. This switch has been associated with telomerase activity, and was likely caused by a mutation that altered the RNA template subunit of telomerase [37]. Telomerase has low fidelity and this may have implications for the binding of telomeric proteins [4]. For the first time in Metazoa, we found Arabidopsis-like telomere (TTTAGGG) ${ }_{n}$ repeats at the ITSs of chromosome 6 in pig cells. Interestingly, we also found that vITSs and bITSs existed together in the interstitial centromeric region in plants belonging to the Hyacinthaceae family, but unlike pig ITSs, they were on several different chromosomes.

Lengths of vITSs vary in different pig cell types and correlate with lengths of TTSs. The lengths of vITSs decrease in pig cells during subculture and increase in iPS cell lines. The length of the vITSs also changed with TTSs in pig primary cells as well as in iPS cell lines. Telomere doublets have often been reported to resemble fragile sites $[43,44]$. These doublets may be associated with telomeric repeat-containing DNA circles (t-circles) [4]. T-SCE detected by $\mathrm{CO}-\mathrm{FISH}$ also can indicate telomere recombination [45]. Telomere doublets and T-SCEs are frequently found in different numbers at the TTSs of various pig cells lines. Telomere doublets and T-SCEs also appear at the interstitial loci in pig telomeres, but are rarely found in normal human and mouse telomeres. The frequency of telomere fragile sites or doublets was found to increase in aphidicolin-induced or TRF1 null mouse cells [43]. TRF1 deficiency leads to telomere fragility and fusion. However, pig telomeres did not show chromosome fusion even after 12-19 passages during subculture, despite being accompanied by an increased frequency in telomere doublets. Natural horsebean and Hyacinthaceae were also found to have telomere doublets in their chromosomes. It has been suggested that the fragility of het-ITSs is related to their propensity to form secondary structures promoting recombination events [46]. In some plants, such as Serissa, Vestia and Cestrum, the typical terminal telomere is not detected, and it has been proposed that they may rely on alternative pathways including t-loops, t-circles, rolling circle replication and recombination for telomere maintenance [4]. Despite completely speculative, pig cells may use part of the telomere regulatory mechanisms in plants to maintain the lengths of their ITSs. The mechanisms underlying the length dynamics of ITSs requires further investigation.

It may be possible to infer the functions of ITSs from the association of heritable diseases with ITS polymorphic variants, in both copy number and sequence [26]. This is one of the most attractive aspects of ITS studies because it could lead to the identification of new and useful markers for genetic linkage studies, forensic applications, and detection of genetic instability in tumors. It is interesting to note that in Hyacinthaceae, the telomeres at the ITSs are remarkably longer than at the TTSs. Therefore, although these plants do not have sufficient telomeres at the TTSs, they can still grow fast, suggesting that the long telomeres at the ITSs may be critical to maintaining cell proliferation. The presence of het-ITSs may relate to chromosomal instability [45]. Indeed, increased ITS loci were observed in gastric carcinoma cells [26] and damaged Chinese hamster cells [46,47]. In addition, plants [4] and immortalized cells without typical terminal telomeres [47], may use similar mechanism for telomere maintenance, for example, the pig ITSs. Further understanding of the mechanisms underlying dynamics and significance of vITSs in pig cells may provide new clues for evolution, chromosomal instability and tumorigenesis.

This work was supported by the National Basic Research Program of China (Grant Nos. 2009CB941000 and 2011CBA01002). We thank Lai LiangXue for providing the Taihu pig fibroblasts.

1 Blackburn E H. Switching and signaling at the telomere. Cell, 2001, 106: 661-673

2 Gomes N M, Shay J W, Wright W E. Telomere biology in Metazoa. FEBS Lett, 2010, 584: 3741-3751

3 Riha K, Shippen D E. Telomere structure, function and maintenance in Arabidopsis. Chromosome Res, 2003, 11: 263-275

4 Fajkus J, Sykorova E, Leitch A R. Telomeres in evolution and evolution of telomeres. Chromosome Res, 2005, 13: 469-479

5 Meyne J, Ratliff R L, Moyzis R K. Conservation of the human telomere sequence (TTAGGG) ${ }_{n}$ among vertebrates. Proc Natl Acad Sci USA, 1989, 86: 7049-7053

6 Meyne J, Baker R J, Hobart H H, et al. Distribution of non-telomeric sites of the (TTAGGG) $)_{n}$ telomeric sequence in vertebrate chromosomes. Chromosoma, 1990, 99: 3-10

7 Lin K W, Yan J. Endings in the middle: current knowledge of interstitial telomeric sequences. Mutat Res, 2008, 658: 95-110

8 Abuin M, Martinez P, Sanchez L. Localization of the repetitive telomeric sequence $\left(\right.$ TTAGGG) ${ }_{n}$ in four salmonid species. Genome, 1996, 39: 1035-1038

9 Garrido-Ramos M A, de la Herran R, Rejon C R, et al. A satellite DNA of the Sparidae family (Pisces, Perciformes) associated with telomeric sequences. Cytogenet Cell Genet, 1998, 83: 3-9

10 Perez J, Moran P, Garcia-Vazquez E. Cloning and physical mapping of Atlantic salmon (Salmo salar L.) telomeric sequences. Heredity, 1999, 82: 409-414

11 Chew J S, Oliveira C, Wright J M, et al. Molecular and cytogenetic analysis of the telomeric (TTAGGG) ${ }_{n}$ repetitive sequences in the Nile tilapia, Oreochromis niloticus (Teleostei: Cichlidae). Chromosoma, 2002, 111: 45-52

12 Nanda I, Schmid M. Localization of the telomeric (TTAGGG) $n$ sequence in chicken (Gallus domesticus) chromosomes. Cytogenet Cell Genet, 1994, 65: 190-193

13 Castiglia R, Gornung E, Corti M. Cytogenetic analyses of chromosomal rearrangements in Mus minutoides/musculoides from NorthWest Zambia through mapping of the telomeric sequence (TTAGGG) and banding techniques. Chromosome Res, 2002, 10: 399-406

14 Yen $\mathrm{C} \mathrm{H}$, Pazik J, Zhang Y, et al. An interstitial telomere array proximal to the distal telomere of mouse chromosome 13. Mamm Genome, 1997, 8: 411-417

15 Bertoni L, Attolini C, Faravelli M, et al. Intrachromosomal telomere-like DNA sequences in Chinese hamster. Mamm Genome, 1996, 7: 853-855

16 Faravelli M, Moralli D, Bertoni L, et al. Two extended arrays of a satellite DNA sequence at the centromere and at the short-arm telomere of Chinese hamster chromosome 5. Cytogenet Cell Genet, 1998, 83: 281-286

17 Ruiz-Herrera A, Garcia F, Azzalin C, et al. Distribution of intrachromosomal telomeric sequences (ITS) on Macaca fascicularis 
(Primates) chromosomes and their implication for chromosome evolution. Hum Genet, 2002, 110: 578-586

18 Uchida W, Matsunaga S, Sugiyama R, et al. Interstitial telomere-like repeats in the Arabidopsis thaliana genome. Genes Genet Syst, 2002, 77: 63-67

19 Dobrzansak M, Kraszewska E, Bucholc M, et al. Molecular cytogenetic analysis of DNA sequences with flanking telomeric repeats in Triticum aestivum cv. Begra. Genome, 2001, 44: 133-136

20 Azzalin C M, Nergadze S G, Giulotto E. Human intrachromosomal telomeric-like repeats: sequence organization and mechanisms of origin. Chromosoma, 2001, 110: 75-82

21 Weber B, Allen L, Magenis R E, et al. Intrachromosomal location of the telomeric repeat (TTAGGG) ${ }_{n}$. Mamm Genome, 1991, 1: 211-216

22 Hastie N D, Allshire R C. Human telomeres: fusion and interstitial sites. Trends Genet, 1989, 5: 326-331

23 JW I J, Baldini A, Ward D C, et al. Origin of human chromosome 2: an ancestral telomere-telomere fusion. Proc Natl Acad Sci USA, 1991, 88: 9051-9055

24 Rossi E, Floridia G, Casali M, et al. Types, stability, and phenotypic consequences of chromosome rearrangements leading to interstitial telomeric sequences. J Med Genet, 1993, 30: 926-931

25 Ruiz-Herrera A, Nergadze S G, Santagostino M, et al. Telomeric repeats far from the ends: mechanisms of origin and role in evolution. Cytogenet Genome Res, 2008, 122: 219-228

26 Mondello C, Pirzio L, Azzalin C M, et al. Instability of interstitial telomeric sequences in the human genome. Genomics, 2000, 68: 111-117

27 Krutilina R I, Oei S, Buchlow G, et al. A negative regulator of telomere-length protein trf1 is associated with interstitial (TTAGGG) blocks in immortal Chinese hamster ovary cells. Biochem Biophys Res Commun, 2001, 280: 471-475

28 Smogorzewska A, van Steensel B, Bianchi A, et al. Control of human telomere length by TRF1 and TRF2. Mol Cell Biol, 2000, 20: 16591668

29 Yang D, Xiong Y, Kim H, et al. Human telomeric proteins occupy selective interstitial sites. Cell Res, 2011, 21: 1013-1027

30 Simonet T, Zaragosi L E, Philippe C, et al. The human TTAGGG repeat factors 1 and 2 bind to a subset of interstitial telomeric sequences and satellite repeats. Cell Res, 2011, 21: 1028-1038

31 de la Sena C, Chowdhary B P, Gustavsson I. Localization of the telomeric (TTAGGG) ${ }_{n}$ sequences in chromosomes of some domestic animals by fluorescence in situ hybridization. Hereditas, 1995, 123: 269-274

32 Takahashi K, Yamanaka S. Induction of pluripotent stem cells from mouse embryonic and adult fibroblast cultures by defined factors.
Cell, 2006, 126: 663-676

33 Herrera E, Samper E, Martin-Caballero J, et al. Disease states associated with telomerase deficiency appear earlier in mice with short telomeres. EMBO J, 1999, 18: 2950-2960

34 Bailey S M, Brenneman M A, Goodwin E H. Frequent recombination in telomeric DNA may extend the proliferative life of telomerase-negative cells. Nucleic Acids Res, 2004, 32: 3743-3751

$35 \mathrm{Gu}$ F, Hindkjaer J, Gustavsson I, et al. A signal of telomeric sequences on porcine chromosome 6q21-q22 detected by primed in situ labelling. Chromosome Res, 1996, 4: 251-252

36 Adams S P, Leitch I J, Bennett M D, et al. Aloe L.-a second plant family without (TTTAGGG) ${ }_{n}$ telomeres. Chromosoma, 2000, 109: 201-205

37 Adams S P, Hartman T P, Lim K Y, et al. Loss and recovery of Arabidopsis-type telomere repeat sequences $5^{\prime}$-(TTTAGGG) ${ }_{n}-3^{\prime}$ in the evolution of a major radiation of flowering plants. Proc Biol Sci, 2001, 268: 1541-1546

38 Weiss H, Scherthan H. Aloe spp.-plants with vertebrate-like telomeric sequences. Chromosome Res, 2002, 10: 155-164

39 Sykorova E, Lim K Y, Kunicka Z, et al. Telomere variability in the monocotyledonous plant order Asparagales. Proc Biol Sci, 2003, 270: 1893-1904

40 Traut W, Szczepanowski M, Vitkova M, et al. The telomere repeat motif of basal Metazoa. Chromosome Res, 2007, 15: 371-382

41 Bailey S M, Williams E S, Cornforth M N, et al. Chromosome orientation fluorescence in situ hybridization or strand-specific FISH. Methods Mol Biol, 2010, 659: 173-183

42 Fradiani P A, Ascenzioni F, Lavitrano M, et al. Telomeres and telomerase activity in pig tissues. Biochimie, 2004, 86: 7-12

43 Sfeir A, Kosiyatrakul S T, Hockemeyer D, et al. Mammalian telomeres resemble fragile sites and require TRF1 for efficient replication. Cell, 2009, 138: 90-103

44 McNees C J, Tejera A M, Martinez P, et al. ATR suppresses telomere fragility and recombination but is dispensable for elongation of short telomeres by telomerase. J Cell Biol, 2010, 188: 639-652

45 Bolzan A D, Bianchi M S. Telomeres, interstitial telomeric repeat sequences, and chromosomal aberrations. Mutat Res, 2006, 612: 189-214

46 Balajee A S, Oh H J, Natarajan A T. Analysis of restriction enzymeinduced chromosome aberrations in the interstitial telomeric repeat sequences of CHO and CHE cells by FISH. Mutat Res, 1994, 307: 307-313

47 Slijepcevic P, Bryant P E. Absence of terminal telomeric FISH signals in chromosomes from immortal Chinese hamster cells. Cytogenet Cell Genet, 1995, 69: 87-89

Open Access This article is distributed under the terms of the Creative Commons Attribution License which permits any use, distribution, and reproduction in any medium, provided the original author(s) and source are credited. 